\title{
An mRNA sequencing analysis of the healing-promoting role of electroacupuncture $a$ in rat skin wound model
}

\author{
Weibin $\mathrm{Du}^{1 \#}$, Guanai Bao ${ }^{2 \#}$, Huahui $\mathrm{Hu}^{1}$, Rongliang Chen ${ }^{1}$, Fuxiang Shen ${ }^{1}$, Renfu Quan ${ }^{1}$, Wenxia $\mathrm{Xu}^{3}$ \\ ${ }^{1}$ Research Institute of Orthopedics, the Affiliated Jiangnan Hospital of Zhejiang Chinese Medical University, Hangzhou, China; ${ }^{2}$ Rehabilitation \\ Department, Cancer Hospital of the University of Chinese Academy of Sciences (Zhejiang Cancer Hospital), Institute of Cancer and Basic Medicine \\ (IBMC), Chinese Academy of Sciences, Hangzhou, China; ${ }^{3}$ Centrol Laboratory, Jinhua Central Hospital, Jinhua, China \\ Contributions: (I) Conception and design: W Du, W Xu; (II) Administrative support: W Du, W Xu; (III) Provision of study materials or patients: \\ None; (IV) Collection and assembly of data: G Bao, H Hu, R Chen; (V) Data analysis and interpretation: W Du , F Shen, R Quan; (VI) Manuscript \\ writing: All authors; (VII) Final approval of manuscript: All authors. \\ \#These authors contributed equally to this work. \\ Correspondence to: Weibin Du. Research Institute of Orthopedics, the Affiliate Jiangnan Hospitial of Zhejiang Chinese Medical University, Hangzhou, \\ China. Email:dwbbdm@163.com; Prof Wenxia Xu. Centrol Laboratory, Jinhua Central Hospital, Jinhua, China. Email: xuwenxia@zju.edu.cn.
}

\begin{abstract}
Background: Many studies have confirmed that electroacupuncture can regulate the body's environment to treat a variety of diseases. However, there are few reports on the mechanism of electroacupuncture therapy for diseases involving skin injury. Transcriptome sequencing can reveal changes in gene expression within cells and the signaling pathways involved. In this study, we used transcriptome sequencing to study the molecular mechanisms by which electroacupuncture promotes the healing of skin lesions.
\end{abstract}

Methods: A total of 10 SD rats were divided into two groups of 5: a control group and an electroacupuncture treatment group. The wound-healing area was compared between the two groups after 3 and 14 days. Then, mRNA sequencing and bioinformatics were used to analyze the changes in gene expression profiles in skin tissue after electroacupuncture stimulation.

Results: (I) The wound area was significantly reduced after 3 and 14 days of electroacupuncture compared with the control group $(\mathrm{P}<0.05)$. (II) There was a total of 694 gene expression changes, 496 of which were upregulated and 198 of which were downregulated. Analysis of variable gene-related signaling pathways by Gene Ontology (GO) and Kyoto Encyclopedia of Genes and Genomes (KEGG), identified immuneinflammatory response, cell proliferation, tissue remodeling, cell metabolism, graft-versus-host disease, antigen processing and presentation, Th17 cell differentiation, cytokine-cytokine receptor interaction, PPAR signaling pathway, Wnt signaling pathway and other signaling pathways were changed.

Conclusions: Electroacupuncture can promote wound repair, as shown by the changes in gene expression profiles during the healing of skin wounds under electroacupuncture. This study provides a scientific basis that deepens the understanding of the mechanism underlying electroacupuncture.

Keywords: Electroacupuncture; skin wound; mRNA sequencing; Gene Ontology (GO); Kyoto Encyclopedia of Genes and Genomes (KEGG)

Submitted Mar 12, 2020. Accepted for publication Jun 10, 2020.

doi: 10.21037/apm-20-626

View this article at: http://dx.doi.org/10.21037/apm-20-626

\section{Introduction}

The repair of the damaged skin tissue is a complex dynamic process, which is regulated by various mechanisms, including the activation of cellular signaling pathways and gene expression $(1,2)$. This dynamic process, which involves the complex progression of wound immune-inflammatory response, tissue re-epithelialization, granulation tissue formation, early vascularization, hair and sweat gland 
tissue regeneration can be summarized into three stages: inflammatory response, cell proliferation, and differentiation, and tissue remodeling $(3,4)$. At present, the standard method for treating skin defects in clinical practice is autologous skin transplantation. Because tissues are derived from the patient, this method requires re-injury, which not only increases the patient's pain, but also entails the risk of complications such as infection and nonunion at the donor site $(5,6)$. Given this, other effective treatment methods have emerged, including new dressings, laser treatment, skin tissue engineering, bioelectric stimulation, and electroacupuncture (7-11).

Acupuncture is a traditional Chinese medical treatment that supplies significant therapeutic effects for many diseases. Electroacupuncture stimulation therapy is a treatment method based on the combination of acupuncture and electric stimulation that enhances the curative effect. It has double the benefits of acupuncture and electric stimulation used alone and is an essential innovation within traditional Chinese medicine. While electroacupuncture treatment research has been carried out in a variety of diseases (12-15), few reports exist concerning the effect and mechanism of electroacupuncture therapy in treating skin lesions (16).

In this study, we used mRNA sequencing and bioinformatics to analyze changes in gene expression profiles in skin tissue after electroacupuncture stimulation. It was found that the expression of 694 genes was altered, and was related to multiple inflammatory pathways, cell proliferation, tissue remodeling, and cell metabolism. It is hoped that these findings will supply a new basis for revealing the molecular mechanism of electroacupuncture therapy for skin damage.

We present the following article in accordance with the ARRIVE reporting checklist (available at http://dx.doi. org/10.21037/apm-20-626).

\section{Methods}

\section{Experimental animals}

A total of $10 \mathrm{SD}$ rats were obtained from the Experimental Animal Center, Zhejiang Chinese Medical University. The purchase and feeding of the rats, along with other animal procedures, were following the guidelines for animal research from the National Institutes of Health and the Committee on Animal Research (17) and was approved by the Experimental Animal Ethics Committee of the Affiliated Jiangnan Hospital of Zhejiang Chinese Medical University (Xiaoshan Traditional Chinese Medical Hospital) (No. XSZYY-2017-016).

\section{Skin resection wound rat model and treatment}

A full-thickness skin resection wound rat model was prepared, as described by Ansell et al. (18). Briefly, SD rats were routinely reared for 1 week, fasted for 12 hours before modeling, made to inhale anesthesia with isoflurane, routinely fixed at five points, modeling area (left side: $2 \mathrm{~cm}$ to the left side of the spine; or right side: right side of the spine $2 \mathrm{~cm}$ ) shearing, hair removal treatment, saline flushing, iodophor disinfection, ethanol deiodination. The central skin of the modeling area was entirely removed by a puncher, and the diameter was $1.5 \mathrm{~cm}$; this was deep in the skin, creating a full-thickness skin wound model. Animal models were divided into a control group and an electroacupuncture treatment group with 5 SD rats in each group. The control group was given a daily treatment of iodophor to prevent wound infection and standard feed. In the electroacupuncture treatment group, electroacupuncture treatment was started on the day of modeling. The center of the wound and 4 points, located $0.5 \mathrm{~cm}$ up and down and left and right from the normal edge of the wound, were selected. A filiform needle $(0.20 \times 13 \mathrm{~mm})$, connected with the Han therapeutic apparatus (Hanfei, Shanghai, China), was then used to perform acupuncture. The negative pole was located in the center of the wound, while the positive pole was located, in turn, on each of the other 4 points. Every point was treated for $10 \mathrm{~min}$, for a total of $40 \mathrm{~min}$ each a day, at a frequency of $2 \mathrm{~Hz}$ and an output current of $1 \mathrm{~mA}$. The rats were given standard feed and iodophor to prevent wound infection.

\section{Determination of wound area}

The wound area on the 3rd and 7th day following injury was measured by a Vernier caliper. The wound area was calculated as follows: long diameter $\times$ short diameter.

\section{RNA extraction and illumine sequencing}

Total RNA was extracted from skin tissues using Trizol reagent (Thermo Fisher, Waltham, MA, USA) following the manufacturer's instruction. The ultraviolet absorbance at 260 and $280 \mathrm{~nm}(260 / 280 \mathrm{~nm})$ was then measured on a Nanodrop 2000 spectrophotometer (Thermo Scientific, Worcester, MA, USA) to determine the concentration and quality of RNA, while the integrity of RNA was detected by LabChip (PerkinElmer, Wellesley, MA, USA). Sequencing analysis was conducted by Biocame Corporation (Wuhan, 
Table 1 Skin wound size of each group at 3 days

\begin{tabular}{lccc}
\hline & Long diameter $(\mathrm{mm})$ & Short diameter $(\mathrm{mm})$ & 127.368 \\
\hline Control 1 & 11.6 & 10.98 & 11.71 \\
Control 2 & 12.33 & 10.44 & 123.2964 \\
Control 3 & 11.81 & 11.04 & 142.1952 \\
Control 4 & 12.88 & 11.01 & 123.6423 \\
Control 5 & 11.23 & 8.41 & 74.5126 \\
Electroacupuncture 1 & 8.86 & 8.54 & 75.579 \\
Electroacupuncture 2 & 8.85 & 7.98 & 68.4684 \\
Electroacupuncture 3 & 8.58 & 8.27 & 70.7912 \\
Electroacupuncture 4 & 8.56 & 7.79 & 68.8636 \\
Electroacupuncture 5 & 8.84 & & \\
\hline
\end{tabular}
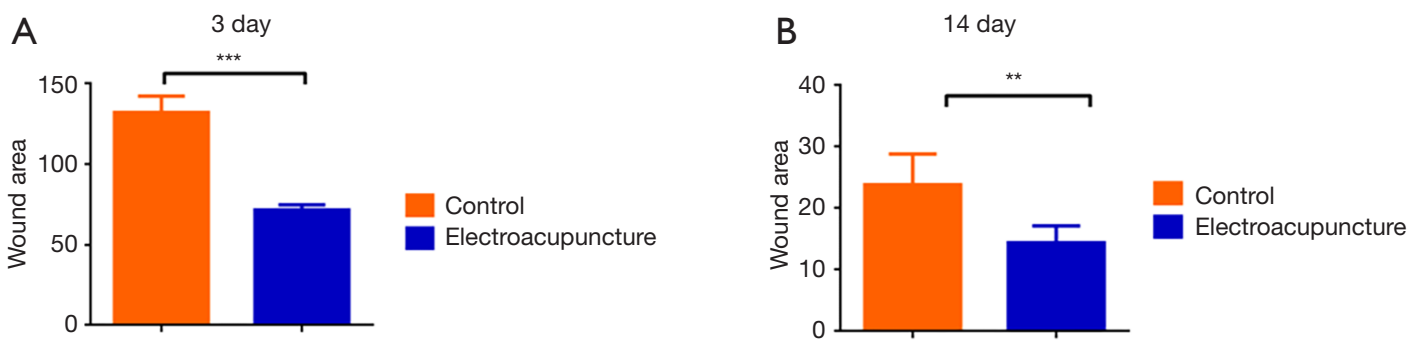

Figure 1 Electroacupuncture stimulation promotes skin wound healing. (A) Histogram of the wound area of the electroacupuncture and control groups after 3 days. (B) Histogram of the wound area of the electroacupuncture and control groups after 14 days. ${ }^{* *} \mathrm{P}<0.01$; ${ }^{* * *} \mathrm{P}<0.001$.

China). Briefly, after the RNA samples were evaluated, the mRNA was enriched with magnetic beads with Oligo (dT), and then a strand-specific cDNA library was constructed. Then, Qubit3.0 was used for quantification (Thermo Scientific, Worcester, MA, USA). Finally, the sample library products were sequenced using an Illumina HiSeqTM 2000.

\section{Bioinformatics analyses of RNA-sequencing data}

GO and KEGG enrichment analysis was performed on the differentially expressed genes found using the cluster profiler package of the R language. The Gene Ontology (GO) terms and Kyoto Encyclopedia of Genes and Genomes (KEGG) pathway filter conditions were considered significantly enriched when their hypergeometric distribution test $\mathrm{P}$ value was less than 0.05 .

\section{Statistical analysis}

The data were presented as means \pm SD (standard deviation). Differences between groups were analyzed using a two-tailed Student's $t$-test. The results were considered statistically significant at $\mathrm{P}<0.05$.

\section{Results}

\section{Electroacupuncture stimulation promotes skin wound bealing}

First, we prepared a full-thickness skin resection wound rat model, as described by Ansell et al. (18). Electroacupuncture was used to treat the skin tissue around the wound. As a result, it was found that the wound area was significantly reduced after 3 days of electroacupuncture compared with the control group (Table 1, Figure 1A). Similarly, the wound 
Table 2 Skin wound size of each group at 14 days

\begin{tabular}{lccc}
\hline & Long diameter $(\mathrm{mm})$ & Short diameter $(\mathrm{mm})$ & Area $\left.(\mathrm{mm})^{2}\right)$ \\
\hline Control 1 & 5.94 & 5.44 & 32.3136 \\
Control 2 & 5.27 & 4.41 & 23.2407 \\
Control 3 & 4.87 & 4.46 & 21.7202 \\
Control 4 & 5.88 & 3.88 & 22.8144 \\
Control 5 & 4.84 & 3.98 & 19.2632 \\
Electroacupuncture 1 & 3.77 & 3.55 & 13.3835 \\
Electroacupuncture 2 & 5.24 & 3.13 & 16.4012 \\
Electroacupuncture 3 & 3.54 & 3.06 & 10.8324 \\
Electroacupuncture 4 & 4.64 & 3.84 & 17.8176 \\
Electroacupuncture 5 & 3.98 & 3.42 & 13.6116 \\
\hline
\end{tabular}

area was significantly smaller than that of the control group after 14 days of electroacupuncture (Table 2, Figure 1B). These results show that electroacupuncture can promote the wound healing of the skin.

\section{Data summary of quality control and sequence alignment of electroacupuncture gene expression profiling}

To clarify how electroacupuncture promotes skin wound healing, we performed gene expression profiling on skin tissue after electroacupuncture. First, we evaluated the quality of the sequenced data. The raw reads obtained by the sequencing had linker sequences and low-quality reads. To ensure the quality of information analysis, we needed to filter the raw reads to obtain clean reads. The latter analysis was based on the clean reads. We used Trimmomatic v.0.36 for quality filtering to ensure that all sequences are above $36 \mathrm{bp}$ (Table 3). Next, we compared the number of reads on the reference genome of the rat. The coincidence rate of the six samples was greater than $93 \%$ (Table 4), suggesting that the quality of clean data was high and could be used for subsequent analysis.

\section{Differentially expressed genes after electroacupuncture treatment}

After statistical analysis, a total of 694 gene expressions were changed after electroacupuncture, 496 of which were upregulated, and 198 of which were downregulated. We used volcano plots and histograms to show these differences (Figure 2). The 30 genes with the most significant upregulation and the 30 genes with the most significant downregulation are listed in Tables 5 and 6 , respectively.

\section{Gene ontology analysis after electroacupuncture treatment}

The GO database explains the role of eukaryotic genes and proteins in cells by creating a set of control words with dynamic forms, including three independent ontologies: biological process (BP), molecular function (MF), and cellular component (CC). Our analysis found that, for biological processes, fatty acid metabolism, immunerelated physiological and pathological processes, and other related genes, were significantly enriched. For the cellular components, cytoplasmic membrane protein complexes, cytoskeletal proteins, lipoproteins, and other related genes were significantly enriched. For molecular function, related genes such as redox reaction and cytokine activity were significantly enriched (Figure 3).

\section{KEGG analysis after electroacupuncture treatment}

In organisms, proteins cannot perform their functions alone, but a series of biochemical reactions are performed by different proteins to perform their biological functions. Therefore, KEGG pathway analysis can help to understand the biological processes or diseases of cells more systematically and comprehensively concerning the mechanism of occurrence. We further analyzed the signaling pathways involved in the differential genes using KEGG. We found a total of 57 significantly altered signaling pathways (Table 7, Figure 4), including immune 
Table 3 clean reads quality statistics

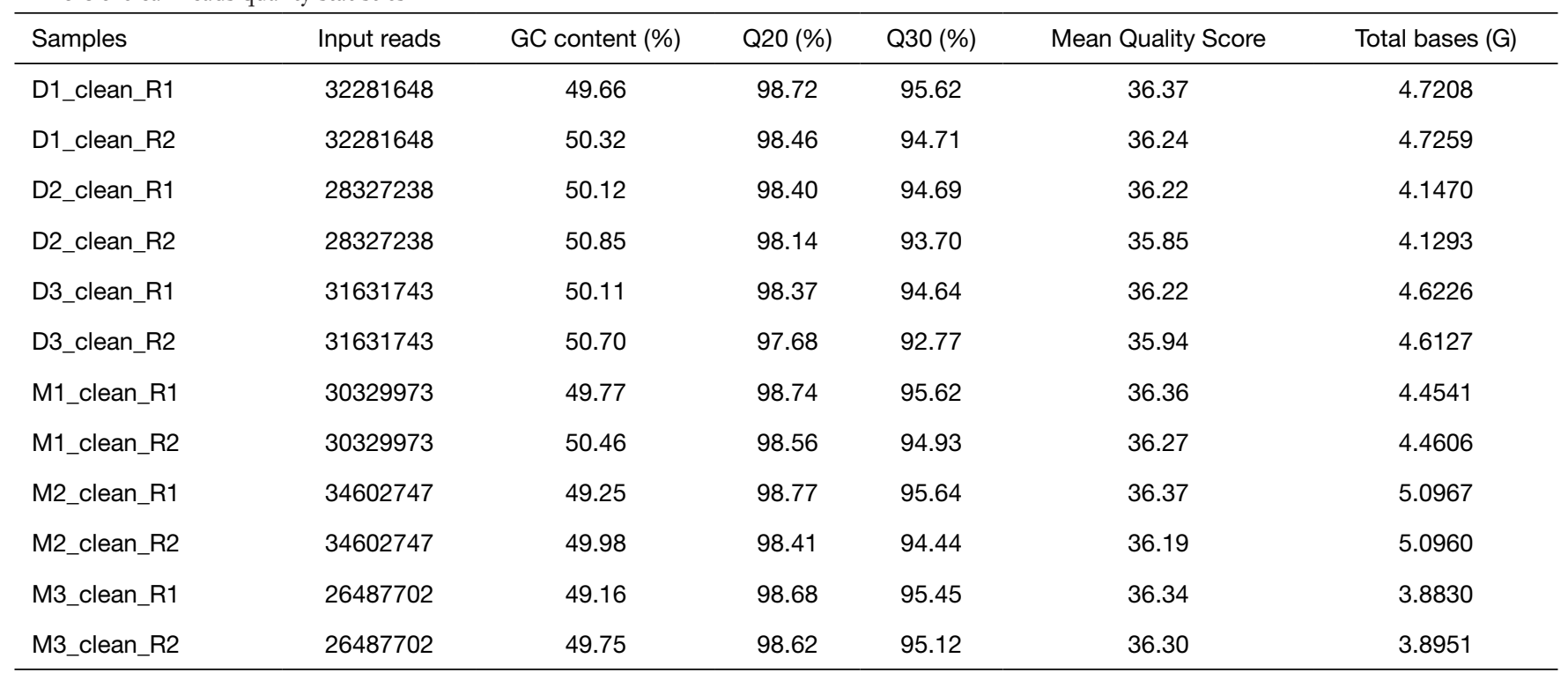

D1-3, samples from electroacupuncture treated rats; M1-3, samples from the control rats. Input reads: Number of filtered reads (clean data). GC content (\%): Percentage of GC content of the sample. Q20 (\%): Bases with a sequencing mass score greater than 20 as a percentage of all bases. Q30 (\%): Bases with a sequencing mass score greater than 30 as a percentage of all bases. Mean Quality Score: Average mass of all bases.

Table 4 Statistical results of reference sequence alignment analysis

\begin{tabular}{|c|c|c|c|c|c|c|c|c|c|c|}
\hline Sample ID & Input reads & $\begin{array}{l}\text { Mapped } \\
\text { reads }\end{array}$ & Non-unique & Unique & Read-1 & Read-2 & $\begin{array}{c}\text { Reads map } \\
\text { to '+' }\end{array}$ & $\begin{array}{c}\text { Reads map } \\
\text { to '-' }\end{array}$ & $\begin{array}{l}\text { Mapped } \\
\text { rate }\end{array}$ & $\begin{array}{l}\text { Concordant pair } \\
\text { alignment rate }\end{array}$ \\
\hline M1 & 60659946 & 58415059 & 7302132 & 51112927 & 25539032 & 25573895 & 25587499 & 25525428 & $96.30 \%$ & $93.59 \%$ \\
\hline M2 & 69205494 & 66880021 & 8032521 & 58847500 & 29407796 & 29439704 & 29452873 & 29394627 & $96.64 \%$ & $94.30 \%$ \\
\hline D1 & 64563296 & 62229318 & 8233841 & 53995477 & 26990521 & 27004956 & 27030726 & 26964751 & $96.38 \%$ & $93.55 \%$ \\
\hline
\end{tabular}

Input reads: Number of filtered reads (clean data). Mapped reads/rate: Can count the number of reads on the reference genome; in general, if there is no contamination and the reference genome is selected appropriately, the percentage of this data is greater than $70 \%$. Non-unique: Quantitative statistics of reads with multiple alignment positions on the reference genome. Unique: Quantitative statistics of reads with unique alignment positions on the reference genome. Read-1, Read-2: The left and right ends of the double-end sequencing read can be compared to the number of reads on the reference genome; Read-1 and Read-2 should be roughly the same. Reads map to '+', Reads map to '-': Quantitative statistics of reads of positive and negative strands of the reference genome. Concordant pair alignment rate: The number of read pairs with the correct relative direction and distance after comparison as a percentage of all read pairs.

responses, cell adhesion, cytokine-receptor interactions, fatty acid metabolism, amino acid metabolism, glucose metabolism, Wnt signaling pathway, PPAR signaling pathway, and others. These results show that the effect of electroacupuncture on skin wound healing involves multiple signaling pathways.

\section{Discussion}

Many clinical and mechanistic studies have confirmed that acupuncture has multidirectional, multilink, multilevel, and multitarget features for the adjustment of the body environment, and has the effect of promoting skin-wound healing (19). Studies have shown that an endogenous 

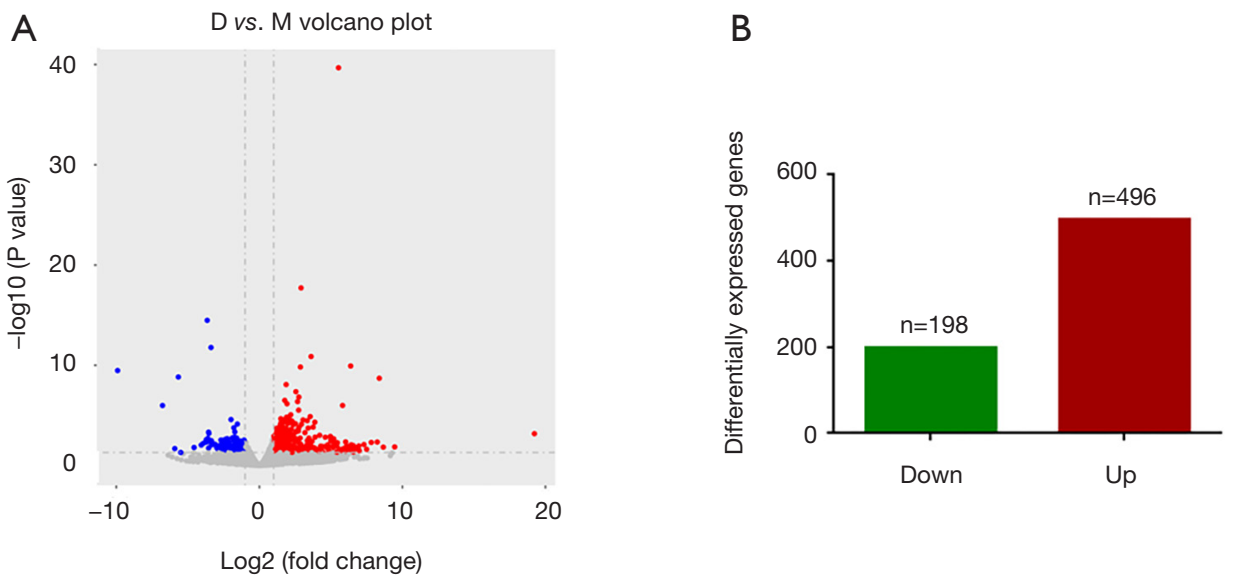

Figure 2 Differentially expressed genes after electroacupuncture treatment. (A) Volcano plots of the differentially expressed genes in the electroacupuncture group compared with the control group. (B) Histogram of differentially expressed genes in the electroacupuncture group compared with the control group.

electric field is generated instantaneously after skin damage, which is very important for wound healing (20). This electric field affects not only epithelial cell metaplasia, but also promotes vascular remodeling (21). Under this electric field, vascular endothelial cells proliferate and migrate, and participate in angiogenesis during injury recovery (22). Therefore, electroacupuncture therapy, which combines acupuncture and an electric field, has become possible for the treatment of skin lesions. In this study, the skin healing of the rat skin lesion model was found to be significantly accelerated by electroacupuncture. Other studies have also reported that electroacupuncture can promote wound healing and tissue reconstruction $(23,24)$. Electroacupuncture also has an improved effect on a variety of diseases, such as female stress urinary incontinence (25), the state of consciousness of patients with acute brain injury (14), Knee arthritis (26), and Crohn's disease (27). Given its versatility, electroacupuncture will be a valuable adjunct in the treatment of various diseases.

Revealing the molecular mechanisms by which electroacupuncture promotes wound healing will help guide clinical practice. Signal pathway regulation processes based on changes in gene expression are decisive for the progression of the disease. Transcriptome sequencing technology facilitates the detection of gene expression profiles under specific conditions (28). The repair of damaged skin tissue includes three overlapping stages of the inflammatory response, cell proliferation, and tissue remodeling, involving multiple signaling pathways and physiological pathological processes (29). The injury itself acts as a signal to initiate the repair mechanism, which promotes local platelet activation and aggregation, and the release of procoagulant factors, chemokines, and growth factors, followed by the release of inflammatory cells such as neutrophils, macrophages, and lymphocytes successively to the wound surface. Locally, each plays a role in wound healing. During wound repair, acupuncture can increase serum immunoglobulin, increase the leukocyte phagocytic index and lymphocyte transformation rate, and enhance the body's anti-infective ability, which can alleviate local prolonged inflammatory response (30,31). Acupuncture can adjust the inflammatory response and immune regulation of the body to the injury in various ways and enhance the tissue repair process. The current research has studied the cholinergic anti-inflammatory (32), BDNFTrkB (33), ERK1/2 signaling (34), and PI3KAkt signaling pathways (35). Our study analyzed the whole genome expression of electroacupuncture in the healing process of skin wounds.

A total of 694 gene expression changes were found, 496 of which were upregulated, and 198 of which were downregulated. Through GO and KEGG analysis, some important genes and related signaling pathways involved in this process were discovered and included immune-inflammatory response, cell proliferation, tissue remodeling, and cell metabolism. Among these were included immune-inflammatory signaling pathways such as graft-versus-host disease, antigen processing and presentation, Th17 cell differentiation, and cytokinecytokine receptor interaction, along with the signaling 
Table 5 The top 30 up-regulated genes in electroacupuncture treatment

\begin{tabular}{|c|c|}
\hline Gene name & log2FoldChange \\
\hline NA & 19.22741904 \\
\hline Cholinergic receptor nicotinic delta subunit & 9.458305939 \\
\hline Cysteine and glycine rich protein 3 & 8.665019697 \\
\hline $40 S$ ribosomal protein $\mathrm{S} 15 \mathrm{a}$-like & 8.382614738 \\
\hline Ribosomal protein S20-like & 8.240717185 \\
\hline Actin, alpha, cardiac muscle 1 & 7.825468831 \\
\hline $\begin{array}{l}\text { S-adenosyl-L-methionine-dependent tRNA } \\
\text { 4-demethylwyosine synthase-like }\end{array}$ & 7.497235776 \\
\hline secreted frizzled-related protein 5 & 7.312931614 \\
\hline NA & 6.988060058 \\
\hline Ninjurin 2 & 6.953798588 \\
\hline fibronectin type III domain containing $3 \mathrm{C} 1$ & 6.862165108 \\
\hline leiomodin-2-like & 6.815364235 \\
\hline INO80 complex subunit B & 6.632455968 \\
\hline Ribosomal protein S20-like & 6.572347946 \\
\hline Contactin 3 & 6.561445851 \\
\hline NA & 6.498230758 \\
\hline NA & 6.417210846 \\
\hline Actin-like 6B & 6.417093771 \\
\hline Aldehyde dehydrogenase family 1 , subfamily $A 7$ & 6.380097819 \\
\hline NA & 6.377126803 \\
\hline Myosin heavy chain 3 & 6.377057103 \\
\hline NA & 6.243881161 \\
\hline Nucleolar protein 8 & 6.18635927 \\
\hline Zinc finger protein 622 & 6.172090834 \\
\hline NA & 6.126515038 \\
\hline V-set and immunoglobulin domain containing 4 & 6.108878669 \\
\hline NA & 6.101202193 \\
\hline Mitotic spindle positioning & 6.097404542 \\
\hline $\begin{array}{l}\text { tRNA (adenine(58)-N(1))-methyltransferase } \\
\text { catalytic subunit TRMT61A }\end{array}$ & 6.030701328 \\
\hline Coiled-coil domain containing 78 & 5.962136591 \\
\hline
\end{tabular}

Table 6 The top 30 down-regulated genes in electroacupuncture treatment

\begin{tabular}{|c|c|}
\hline Gene name & log2FoldChange \\
\hline NA & -9.912670007 \\
\hline Solute carrier family 40 member 1 & -6.77097968 \\
\hline NA & -5.913759087 \\
\hline NA & -5.664684773 \\
\hline NA & -5.498714468 \\
\hline NA & -4.564953962 \\
\hline Zinc finger, SWIM-type containing 8 & -4.061554952 \\
\hline $\begin{array}{l}\text { Potassium channel subfamily } \mathrm{K} \\
\text { member } 6 \text {-like }\end{array}$ & -3.940450148 \\
\hline Ribosomal protein S15A-like 4 & -3.722423621 \\
\hline Selenium binding protein 1 & -3.686797569 \\
\hline $\begin{array}{l}\text { Family with sequence similarity } 111, \\
\text { member A }\end{array}$ & -3.646212036 \\
\hline Ribonuclease K6-like & -3.60616759 \\
\hline Ring finger protein 182 & -3.604859799 \\
\hline Ribosomal L1 domain containing 1-like 1 & -3.551409253 \\
\hline $\begin{array}{l}\text { Signal peptide, CUB domain and EGF like } \\
\text { domain containing } 3\end{array}$ & -3.549708769 \\
\hline NA & -3.530317206 \\
\hline NA & -3.380613979 \\
\hline NA & -3.356546631 \\
\hline NA & -3.340436425 \\
\hline G protein-coupled receptor 156 & -3.278747947 \\
\hline Keratin 39 & -3.164058043 \\
\hline Desmoglein 4 & -3.065752115 \\
\hline Synaptotagmin 7 & -2.991379393 \\
\hline GC-rich promoter binding protein 1-like 2 & -2.964846903 \\
\hline NA & -2.915822674 \\
\hline $\begin{array}{l}\text { Calcium homeostasis modulator family } \\
\text { member } 4\end{array}$ & -2.889600213 \\
\hline NA & -2.883201329 \\
\hline Keratin associated protein 2-4-like & -2.751921907 \\
\hline Keratin-associated protein 4-6-like & -2.73900119 \\
\hline NA & -2.704128242 \\
\hline
\end{tabular}




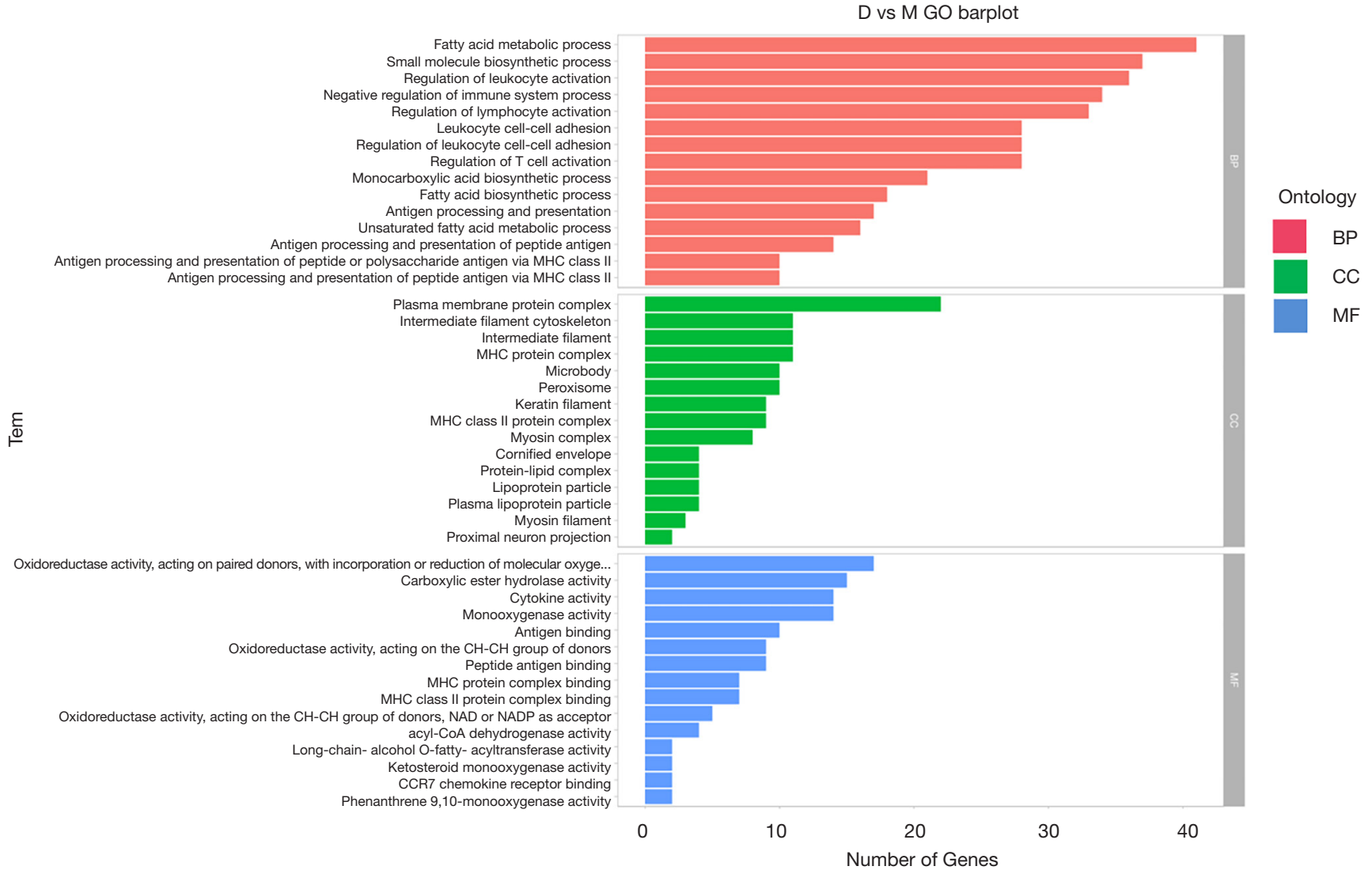

Figure 3 GO analysis histogram of differentially expressed genes in the electroacupuncture group compared with the control group. GO, Gene Ontology.

Table 7 List of significant enrichment KEGG pathway

\begin{tabular}{|c|c|c|c|}
\hline KEGG_ID & Description & $P$ value & Gene ID \\
\hline rno04514 & Cell adhesion molecules (CAMs) & $2.58 \mathrm{E}-08$ & $\begin{array}{l}\text { Cldn2/Nlgn2/RT1-M2/RT1-A1/Cldn5/RT1-M6-1/RT1-Bb/RT1-DOa/ } \\
\text { RT1-CE5/Itga8/RT1-DMa/Cdh2/Cd86/RT1-CE7/Cd99/RT1-DMb/ } \\
\text { RT1-Da/RT1-Db1/RT1-Ba/Ntng1/RT1-DOb }\end{array}$ \\
\hline rno04940 & Type I diabetes mellitus & 4.79E-08 & $\begin{array}{l}\text { RT1-A1/RT1-M6-1/RT1-Bb/RT1-DOa/RT1-CE5/RT1-CE7/RT1-DMb/ } \\
\text { RT1-Db1/RT1-M2/RT1-DMa/Cd86/RT1-Da/RT1-Ba/RT1-DOb }\end{array}$ \\
\hline rno04612 & $\begin{array}{l}\text { Antigen processing and } \\
\text { presentation }\end{array}$ & $1.53 \mathrm{E}-07$ & $\begin{array}{l}\mathrm{RT} 1-\mathrm{DOb} / \mathrm{RT} 1-\mathrm{A} 1 / \mathrm{RT} 1-\mathrm{M} 6-1 / \mathrm{RT} 1-\mathrm{Bb} / \mathrm{RT} 1-\mathrm{DOa} / \mathrm{RT} 1-\mathrm{CE} 5 / \mathrm{RT} 1-\mathrm{CE} 7 / \\
\mathrm{Cd} 74 / \mathrm{RT} 1-\mathrm{DMb} / \mathrm{RT} 1-\mathrm{Db} 1 / \mathrm{RT} 1-\mathrm{M} 2 / \mathrm{RT} 1-\mathrm{Da} / \mathrm{RT} 1-\mathrm{DMa} / \mathrm{Ciita} / \mathrm{RT} 1-\mathrm{Ba}\end{array}$ \\
\hline
\end{tabular}

Table 7 (continued) 
Table 7 (continued)

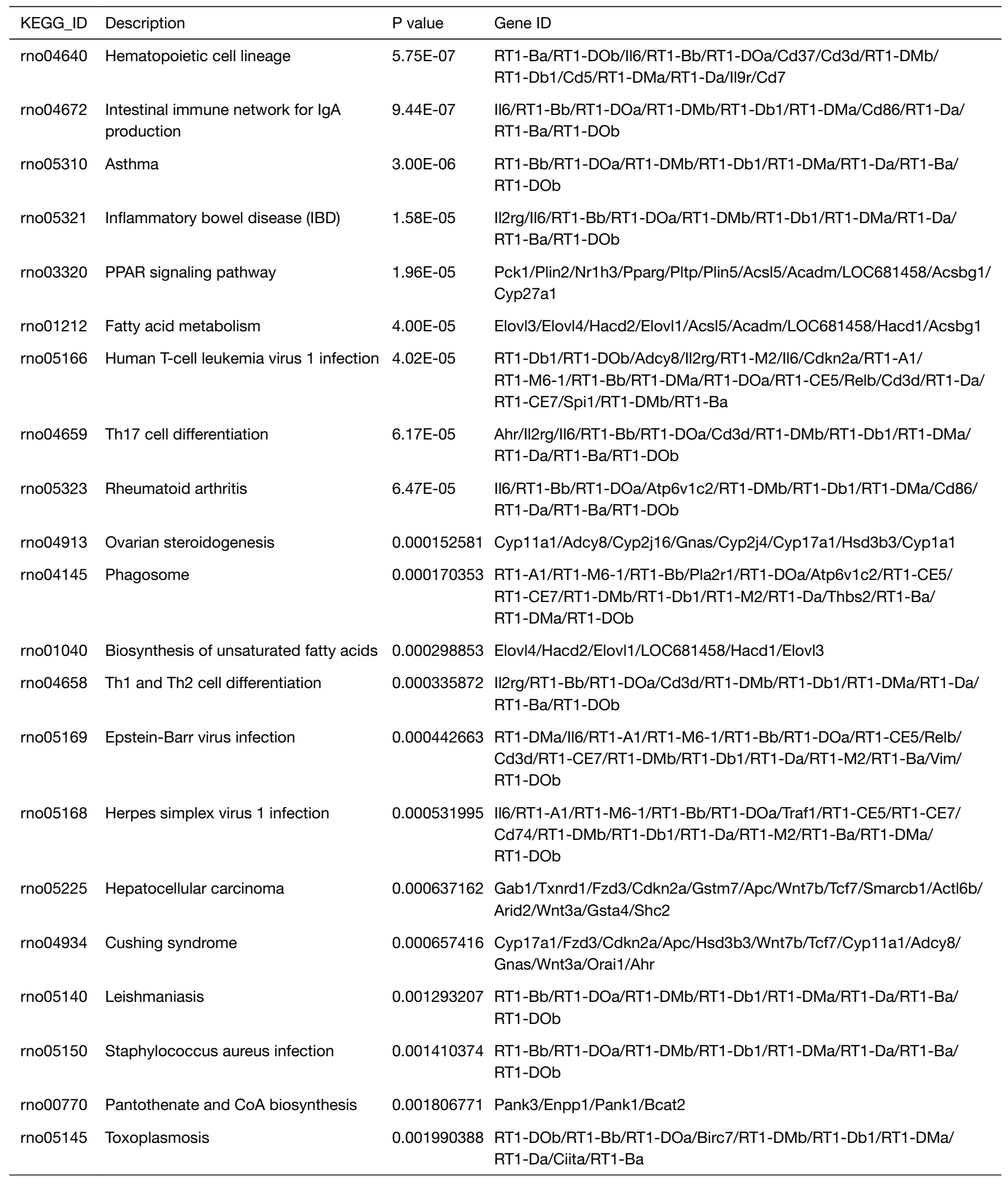

Table 7 (continued) 
Table 7 (continued)

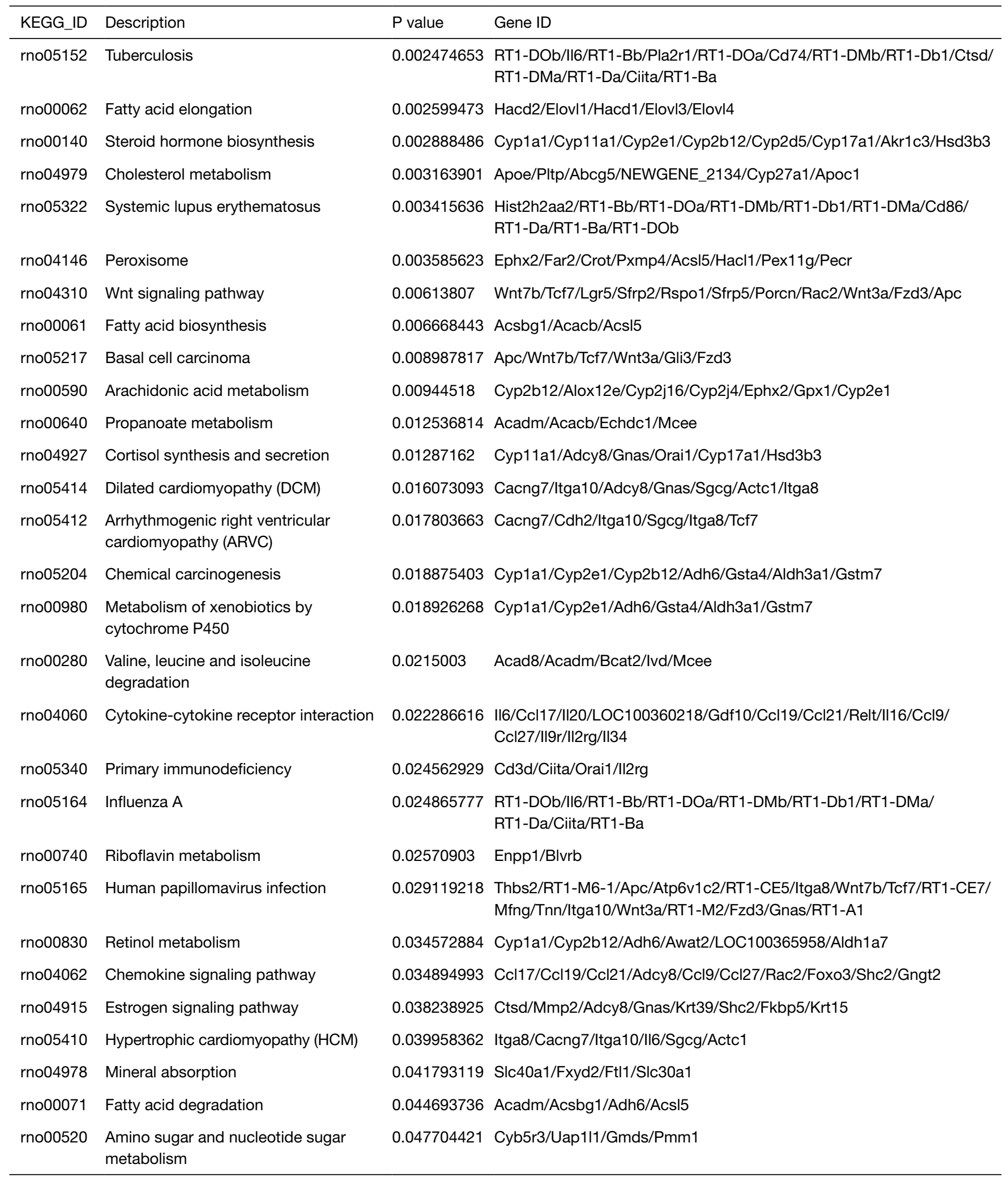

KEGG, Kyoto Encyclopedia of Genes and Genomes. 


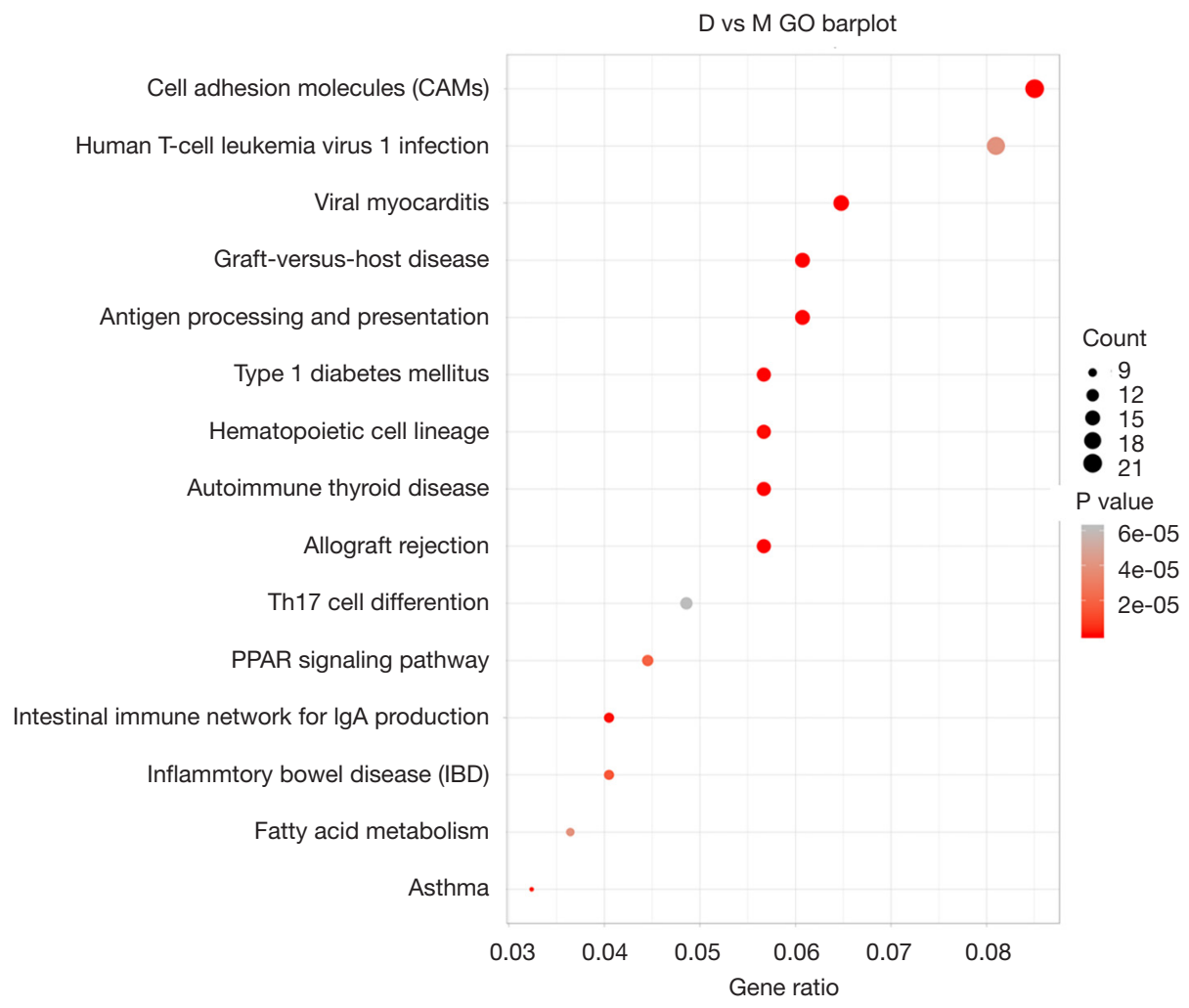

Figure 4 Scatter diagram of KEGG pathway enrichment. KEGG, Kyoto Encyclopedia of Genes and Genomes.

pathways of cell proliferation, such as PPAR signaling pathway, Wnt signaling pathway, and tissue remodeling signaling pathways. Cell adhesion molecules and the signaling pathways involved in cell metabolism included fatty acid, an amino sugar, and nucleotide sugar metabolism. These data indicate that the changes of the signal network in the skin tissue under the action of electroacupuncture involve many critical signaling pathways related to skin healing. The metabolic, immune-inflammatory and growth-promoting pathways have been over-activated in the skin wound healing of the middle-aged mice, reported in previous study (36). Omega-3 polyunsaturated fatty acids (PUFAs) can promote wound healing by affecting the synthesis and activity of cytokines (37). Heparinbinding EGF-like Growth Factor (HB-EGF), a member of the EGF family, accelerates Keratinocyte migration and skin wound Healing (38). Study also demonstrated that angiotensin II plays an important role in skin wound healing via accelerating keratinocyte and fibroblast migration in a process mediated by HB-EGF shedding (39). What's more, chemokine (TARC)/CC chemokine ligand 17 (CCL17) accelerates wound healing, mainly by enhancing fibroblast migration, and possibly by increasing $\mathrm{NGF}(+)$ lymphocytes and mast cells, which have independently been reported to enhance wound healing (40). In our sequencing results, the expression level of CCL17 was promoted, which may contribute to skin healing. The Wnt signaling pathway is closely related to skin growth and development and participates in the regulation of the wound healing process, which in turn plays a vital role in regulating cell proliferation, differentiation, migration, and apoptosis $(41,42)$. In our sequencing results, the expression level of Wnt7a, a gene that activates the Wnt signaling pathway, was significantly upregulated, while the expression level of the significant negative regulator, adenomatous polyposis coli (APC), was significantly downregulated. This means that the Wnt signaling pathway is indeed activated under electroacupuncture stimulation, thereby promoting skin healing. Peroxisome proliferator-activated receptor (PPAR) $\beta / \delta$, which is known to be involved for example in lipid catabolism, glucose homeostasis, inflammation, survival, proliferation, differentiation, as well as mammalian regeneration of the skin, bone and liver-appears to be a promising target to promote mammalian regeneration (43). 
The analysis of wound healing in PPAR $\beta / \delta$ knockout mice revealed that $\mathrm{PPAR} \beta / \delta$ is required during skin healing for keratinocyte proliferation resulting in a delay in healing by two to three days (44). In our sequencing results, the PPAR signaling pathway was activated, which means it participated in wound healing. Studies that examined the genome-wide expression of electroacupuncture-treated rat myocardial ischemia-reperfusion models (45) found that the signaling pathways involved included immune inflammation, and cell proliferation and apoptosis-how electroacupuncture signals initiate the gene expression regulation process warrants further research. Ultimately, the regulation of electrophysiology and the pathology of electricity is a comprehensive process involving multiple signaling pathways, and this complexity underlies the distinction between the use of electroacupuncture treatment and other monotherapy.

In summary, our study demonstrated electroacupuncture's wound-repairing effects and identified the accompanying changes in gene expression profiles. It is hoped that these findings can provide a scientific basis for further insight into the mechanism of electroacupuncture.

\section{Acknowledgments}

Funding: This work was supported by National Natural Science Foundation of China (no.81904053), the Plan Guide Project of Hangzhou Technology Department (no.20171226Y93, 20171226Y96), the Plan Project of Hangzhou Health Science and Technology Department (no.2018B030), and the Great Technology Capture Project of Hangzhou Xiaoshan District (no.2017208, 2017214).

\section{Footnote}

Reporting Checklist: The authors have completed the ARRIVE reporting checklist. Available at http://dx.doi. org/10.21037/apm-20-626

Data Sharing Statement: Available at http://dx.doi. org/10.21037/apm-20-626

Conflicts of Interest: All authors have completed the ICMJE uniform disclosure form (available at http://dx.doi. org/10.21037/apm-20-626). The authors have no conflicts of interest to declare.

Ethical Statement: The authors are accountable for all aspects of the work in ensuring that questions related to the accuracy or integrity of any part of the work are appropriately investigated and resolved. The experimental protocol was set up according to the guidelines for animal research from the National Institutes of Health and the Committee on Animal Research and was approved by the Experimental Animal Ethics Committee of the Affiliated Jiangnan Hospital of Zhejiang Chinese Medical University (Xiaoshan Traditional Chinese Medical Hospital) (No. XSZYY-2017-016).

Open Access Statement: This is an Open Access article distributed in accordance with the Creative Commons Attribution-NonCommercial-NoDerivs 4.0 International License (CC BY-NC-ND 4.0), which permits the noncommercial replication and distribution of the article with the strict proviso that no changes or edits are made and the original work is properly cited (including links to both the formal publication through the relevant DOI and the license). See: https://creativecommons.org/licenses/by-nc-nd/4.0/.

\section{References}

1. Nourian Dehkordi A, Mirahmadi Babaheydari F, Chehelgerdi $M$, et al. Skin tissue engineering: wound healing based on stem-cell-based therapeutic strategies. Stem Cell Res Ther 2019;10:111.

2. Rosinczuk J, Taradaj J, Dymarek R, et al. Mechanoregulation of Wound Healing and Skin Homeostasis. Biomed Res Int 2016;2016:3943481.

3. Casano AM, Sixt M. A Fat Lot of Good for Wound Healing. Dev Cell 2018;44:405-6.

4. Ennis WJ, Lee C, Gellada K, et al. Advanced Technologies to Improve Wound Healing: Electrical Stimulation, Vibration Therapy, and Ultrasound-What Is the Evidence? Plast Reconstr Surg 2016;138:94s-104s.

5. Ud-Din S, Bayat A. Electrical Stimulation and Cutaneous Wound Healing: A Review of Clinical Evidence. Healthcare (Basel) 2014;2:445-67.

6. Quan R, Du W, Zheng X, et al. VEGF165 induces differentiation of hair follicle stem cells into endothelial cells and plays a role in in vivo angiogenesis. J Cell Mol Med 2017;21:1593-604.

7. Zhang T, Liu F, Tian W. Advance of new dressings for promoting skin wound healing. Sheng Wu Yi Xue Gong Cheng Xue Za Zhi 2019;36:1055-9.

8. Nguyen J, Chapman LW, Korta DZ, et al. Laser treatment of cutaneous angiokeratomas: A systematic review. 
Dermatol Ther 2017;30. doi: 10.1111/dth.12558.

9. Chaudhari AA, Vig K, Baganizi DR, et al. Future Prospects for Scaffolding Methods and Biomaterials in Skin Tissue Engineering: A Review. Int J Mol Sci 2016;17:1974.

10. Kai H, Yamauchi T, Ogawa Y, et al. Accelerated Wound Healing on Skin by Electrical Stimulation with a Bioelectric Plaster. Adv Healthc Mater 2017;6. doi: 10.1002/adhm.201700465.

11. Wang Y, Fu Y, Zhang L, et al. Acupuncture Needling, Electroacupuncture, and Fire Needling Improve Imiquimod-Induced Psoriasis-Like Skin Lesions through Reducing Local Inflammatory Responses. Evid Based Complement Alternat Med 2019;2019:4706865.

12. Yang M, Wan Y, Jiang X, et al. Electro-Acupuncture Promotes Accumulation of Paclitaxel by Altering Tumor Microvasculature and Microenvironment in Breast Cancer of Mice. Front Oncol 2019;9:576.

13. Li M, Wang L, Xu N, et al. Effect of electro-acupuncture on lateralization of the human swallowing motor cortex excitability in healthy subjects: study protocol for a singleblind, randomized controlled trial. Trials 2019;20:180.

14. Liu J, Xue X, Wu Y, et al. Efficacy and safety of electroacupuncture treatment in improving the consciousness of patients with traumatic brain injury: study protocol for a randomized controlled trial. Trials 2018;19:296.

15. Zhang R, Andersen AH, Hardy PA, et al. Objectively measuring effects of electro-acupuncture in parkinsonian rhesus monkeys. Brain Res 2018;1678:12-9.

16. Walton EW. Electroacupuncture: applications in the treatment of chronic nonhealing wounds. Adv Skin Wound Care 2013;26:495-7.

17. National Research Council Committee for the Update of the Guide for the C, Use of Laboratory A. The National Academies Collection: Reports funded by National Institutes of Health. Guide for the Care and Use of Laboratory Animals. Washington (DC): National Academies Press (US) Copyright (C) 2011, National Academy of Sciences; 2011.

18. Ansell DM, Holden KA, Hardman MJ. Animal models of wound repair: Are they cutting it? Exp Dermatol 2012;21:581-5.

19. Papantonio C. Alternative medicine and wound healing. Ostomy Wound Manage 1998;44:44-6, 8, 50 passim.

20. Martin-Granados C, McCaig CD. Harnessing the Electric Spark of Life to Cure Skin Wounds. Adv Wound Care (New Rochelle) 2014;3:127-38.

21. Zhao Z, Qin L, Reid B, et al. Directing migration of endothelial progenitor cells with applied DC electric fields. Stem Cell Res 2012;8:38-48.

22. Ud-Din S, Sebastian A, Giddings P, et al. Angiogenesis is induced and wound size is reduced by electrical stimulation in an acute wound healing model in human skin. PLoS One 2015;10:e0124502.

23. Foell J. Acupuncture as add-on treatment in the management of a patient with ecthyma gangrenosum. Acupunct Med 2012;30:60-2.

24. Sumano H, Mateos G. The use of acupuncture-like electrical stimulation for wound healing of lesions unresponsive to conventional treatment. Am J Acupunct 1999;27:5-14.

25. Liu Z, Liu Y, Xu H, et al. Effect of Electroacupuncture on Urinary Leakage Among Women With Stress Urinary Incontinence: A Randomized Clinical Trial. Jama 2017;317:2493-501.

26. Chen N, Wang J, Mucelli A, et al. Electro-Acupuncture is Beneficial for Knee Osteoarthritis: The Evidence from Meta-Analysis of Randomized Controlled Trials. Am J Chin Med 2017;45:965-85.

27. Bao C, Liu P, Liu H, et al. Different brain responses to electro-acupuncture and moxibustion treatment in patients with Crohn's disease. Sci Rep 2016;6:36636.

28. Wu Y, Zhao J, Dong S, et al. Whole-exome and RNA sequencing reveal novel insights into the pathogenesis of HPV associated cervical cancer. Cancer Biomark 2019;25:341-50.

29. Komarcević A. The modern approach to wound treatment. Med Pregl 2000;53:363-8.

30. Yiu EM, Chan KM, Li NY, et al. Wound-healing effect of acupuncture for treating phonotraumatic vocal pathologies: A cytokine study. Laryngoscope 2016;126:E18-22.

31. Lee JA, Jeong HJ, Park HJ, et al. Acupuncture accelerates wound healing in burn-injured mice. Burns 2011;37:117-25.

32. Zhang XF, Xiang SY, Geng WY, et al. Electro-acupuncture regulates the cholinergic anti-inflammatory pathway in a rat model of chronic obstructive pulmonary disease. J Integr Med 2018;16:418-26.

33. Tu WZ, Li SS, Jiang X, et al. Effect of electro-acupuncture on the BDNF-TrkB pathway in the spinal cord of CCI rats. Int J Mol Med 2018;41:3307-15.

34. Zhang Y, Yu JB, Luo XQ, et al. Effect of ERK1/2 signaling pathway in electro-acupuncture mediated up-regulation of heme oxygenase-1 in lungs of rabbits with endotoxic shock. Med Sci Monit 2014;20:1452-60.

35. Xue X, You Y, Tao J, et al. Electro-acupuncture at points of Zusanli and Quchi exerts anti-apoptotic effect through the modulation of PI3K/Akt signaling pathway. Neurosci 
Lett 2014;558:14-9.

36. Yanai H, Lumenta DB, Vierlinger K, et al. Middle age has a significant impact on gene expression during skin wound healing in male mice. Biogerontology 2016;17:763-70.

37. Huang TH, Wang PW, Yang SC, et al. Cosmetic and Therapeutic Applications of Fish Oil's Fatty Acids on the Skin. Mar Drugs 2018;16:256.

38. Shirakata Y, Kimura R, Nanba D, et al. Heparin-binding EGF-like growth factor accelerates keratinocyte migration and skin wound healing. J Cell Sci 2005;118:2363-70.

39. Yahata Y, Shirakata Y, Tokumaru S, et al. A novel function of angiotensin II in skin wound healing. Induction of fibroblast and keratinocyte migration by angiotensin II via heparin-binding epidermal growth factor (EGF)-like growth factor-mediated EGF receptor transactivation. J Biol Chem 2006;281:13209-16.

40. Kato T, Saeki H, Tsunemi Y, et al. Thymus and activationregulated chemokine (TARC)/CC chemokine ligand (CCL) 17 accelerates wound healing by enhancing

Cite this article as: $\mathrm{Du}$ W, Bao G, Hu H, Chen R, Shen F, Quan R, Xu W. An mRNA sequencing analysis of the healingpromoting role of electroacupuncture a in rat skin wound model. Ann Palliat Med 2020;9(4):1462-1475. doi: 10.21037/ apm-20-626 fibroblast migration. Exp Dermatol 2011;20:669-74.

41. Okuse T, Chiba T, Katsuumi I, et al. Differential expression and localization of WNTs in an animal model of skin wound healing. Wound Repair Regen 2005;13:491-7.

42. Wang W, Yan X, Lin Y, et al. Wnt7a promotes wound healing by regulation of angiogenesis and inflammation: Issues on diabetes and obesity. J Dermatol Sci 2018. [Epub ahead of print].

43. Magadum A, Engel FB. PPAR $\beta / \delta$ : Linking Metabolism to Regeneration. Int J Mol Sci 2018;19:2013.

44. Michalik L, Desvergne B, Tan NS, et al. Impaired skin wound healing in peroxisome proliferator-activated receptor (PPAR)alpha and PPARbeta mutant mice. J Cell Biol 2001;154:799-814.

45. Huang Y, Lu SF, Hu CJ, et al. Electro-acupuncture at Neiguan pretreatment alters genome-wide gene expressions and protects rat myocardium against ischemiareperfusion. Molecules 2014;19:16158-78. 